\title{
The 2MASS Red AGN Survey
}

\author{
R.M. Cutri, B.O. Nelson \\ Infrared Processing and Analysis Center/California Institute of \\ Technology, MS 100-22, Pasadena, CA 91125, email: \\ roc@ipac.caltech.edu,nelson@ipac.caltech.edu \\ Paul J. Francis \\ The Australian National University, Canberra, ACT 0200, Australia, \\ email: pfrancis@mso.anu.edu.au \\ Paul S. Smith \\ Univ. of Arizona, Steward Obs., Tucson, AZ 85721, USA, email: \\ psmith@as.arizona.edu
}

\begin{abstract}
The Two Micron All Sky Survey (2MASS) provides an unprecedented, uniform photometric data set for large samples of AGN discovered at other wavelengths, and also forms the basis to search for previously unknown, obscured AGN. We present the results of a highly efficient near infrared color-based AGN survey using 2MASS that has already discovered 485 new, red AGN and QSOs. The extrapolated surface density of the 2MASS red AGN is $\sim 0.57 \mathrm{deg}^{-2}$ for $K_{s} \leq 15.5 \mathrm{mag}$. The ratio of Type 1 to Type 2 AGN among the newly discovered objects is $4: 1$, similar to proportions found in X-ray and deep ISO surveys, but the inverse of that found in IRAS surveys. The median redshift of the the new 2MASS red AGN sample is $\mathrm{z}=0.22$, and all but three have $\mathrm{z}<0.7$. The color distribution, polarization and X-ray properties data suggest that most of the 2MASS-discovered AGN are red because of obscuration by dust in and around their nuclei.
\end{abstract}

\section{Introduction}

Much of the current wisdom about the distribution, energetics and evolution of Active Galactic Nuclei (AGN) and Quasi-stellar Objects (QSOs) is based on samples of objects found in UV-excess or optical color surveys. While it has been known for at least 30 years that some AGN are partially obscured by dust in and around their nuclei (e.g. Mrk 231, NGC 1068), there has been increasing evidence in the past decade that large numbers of AGN have remained hidden from traditional UV/optical surveys because of dust obscuration. Infrared and radio surveys, less affected by dust extinction, suggest that between $50 \%$ and $80 \%$ of all AGN have been missed in the existing short wavelength surveys (Low et al. 1988; Webster et al. 1995; Francis et al 1999). The existence of a large population of obscured AGN has also been proposed to account for at least part 
of the hard X-ray background (e.g. Comastri et al. 1995; Gilli et al. 2001), and recent recent deep Chandra observations may be revealing this population (Mushotzky et al. 2000).

Seemingly at odds with the findings of the radio, infrared and X-ray surveys are the results of recent deeper optical-color surveys that claim to be no more than 10-20AGN (e.g. Meyer et al. 2001; Ivezic et al. this volume). We use data from the highly uniform Two Micron All Sky Survey (2MASS; Skrutskie et al. 1997) to address these apparently disparate results by conducting a large-scale search for previously unknown, red AGN. The 2MASS red AGN survey uses a simple, highly efficient color-selection criterion that is well-suited to identify relatively low redshift AGN. The objective of this search is not to find all AGN detected by 2MASS, but rather to test for the existence of a population of extremely red AGN. We present here the preliminary results of this Survey.

\section{Red AGN Search Criteria}

A characteristic of known AGN is that their near infrared colors are redder than most foreground stars and normal galaxies (e.g. Rieke 1978; Neugebauer et al. 1987). Obscured AGN will stand out from foreground stars and low redshift galaxies with even greater contrast. Because 2MASS provides uniform photometry in the $\mathrm{J}(1.25 \mu \mathrm{m}), \mathrm{H}(1.65 \mu \mathrm{m})$ and $K_{s}(2.17 \mu \mathrm{m})$ near infrared bandpasses over the full-sky, we tuned a color-selection criterion to best discriminate reddened AGN from UV/optically-selected ones using the measurements of large samples of known AGN. 2MASS detects $100 \%, 99 \%$ and $75 \%$ of the PG QSOs (Schmidt \& Green 1986), the Hamburg/ESO QSOs (Wisotzki et al. 2000), and the LBQS QSOs (Hewitt et al. 1995), respectively, in all three near-infrared bands. $95 \%$ of the combined set of AGN from these optical/UV surveys have $J-K_{s}<2.0$. Therefore, we employ this color limit as the primary selection criterion to identify obscured AGN.

We selected candidates from the 2MASS Point Source Working Databases by requiring a detection in all three 2MASS bands and $J-K_{s}>2.0$. To minimize contamination by foreground AGB stars, which also occupy this part of color space, the search was limited to the $|b|>30^{\circ}$ sky and excluded $\sim 170 \mathrm{deg}^{2}$ covering the Large and Small Magellanic Clouds. There are 16,977 unique candidates in an effective area of $\sim 20,400 \mathrm{deg}^{2}\left(0.83\right.$ candidates $\left.\mathrm{deg}^{-2}\right)$ that satisfy these criteria. The brightness and color distribution of the color-selected candidates are shown in Figure 1. Less than $5 \%$ of the candidates are previously identified objects, and $\leq 1 \%$ are previously known AGN. Virtually all the candidates with $K_{s} \leq 11 \mathrm{mag}$ are high latitude Galactic AGB and carbon stars.

No requirement was imposed on the either the near-infrared or optical morphology of the candidates. Many sources appear slightly extended on either the 2MASS images or Palomar and ESO sky survey prints. Dilution of the nuclear light by starlight from the host galaxy may influence the observed infrared and optical color properties of the sample. The rest-frame 2MASS colors of galaxies (Jarrett et al. 2000) are significantly bluer than our color-selection criterion, so the lowest redshift, lowest luminosity AGN are missed by our sample. The galaxy k-correction shifts $J-K_{s}$ colors to the red with increasing redshift, though, and by redshift $\mathrm{z} \sim 0.4-0.5$, some "normal" galaxies may begin to shift into our 


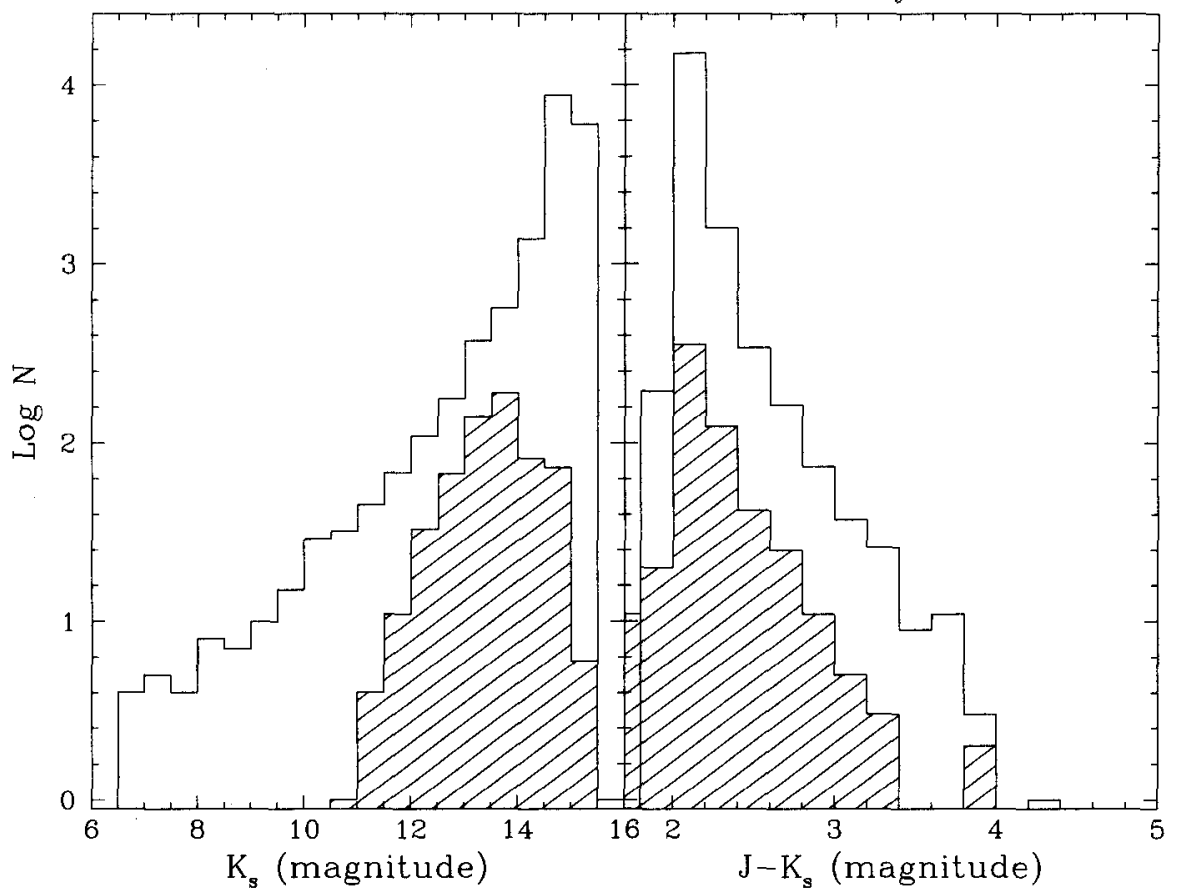

Figure 1. (left) The $K_{s}$ brightness distribution of the 16,997 colorselected red AGN candidates selected from the 2MASS Point Source Working Databases. (right) The $J-K_{s}$ color distribution of 2MASS red AGN candidates. In both panels, the distribution of sources with follow-up optical spectroscopy is shown in the shaded regions.

color window. An $L^{*}$ galaxy at $z=0.4$ will be nearly 2 magnitudes below the 2MASS $K_{s}$ detection limit, though, so only the brightest cluster galaxies will be marginally detectable at that distance $\left(K_{s} \sim 15.0 \mathrm{mag}\right.$; Brough et al. 2001). We expect the contamination from non-active galaxies to be relatively small in our sample, but it may increase towards the faintest $K_{s}$ magnitudes.

Dilution by starlight from the host galaxy will make the optical colors of an AGN appear redder, and less AGN-like. Thus, it would not be unexpected to find galaxies harboring extinguished active nuclei that exhibit relatively normal galaxy-like optical colors. The active nucleus would be more apparent at longer wavelengths where the affect of obscuration is reduced.

\section{Source Classification and Properties of the 2MASS AGN}

Optical spectroscopy has now been carried out for 704 (4.1\%) of the red AGN candidates at a variety of telescopes. Object classification and redshift measurements were made from the optical spectra: $384(44 \%)$ of the objects are confirmed broad-line Type 1 AGN (Sy1/QSO), 101 (14\%) are narrow-line Type 2 AGN (Sy2/LINER), 108 (15\%) are weak emission line or normal galaxies, and $71(10 \%)$ are carbon and L-dwarf stars. Approximately $5 \%$ of the candidates 
could not be identified because of insufficient signal-to-noise ratio in their optical spectra. Type $1 \mathrm{AGN}$ are taken to be any object with broad emission-line components, and thus contain all of the intermediate AGN classes. We make no luminosity-based distinction between Sy 1 and QSO. The ratio of Type 1 and Type 2 (no broad component) AGN is $\sim 4: 1$.

Figure 1 also shows the brightness and color distributions of the sources that have optical spectroscopy. The sampling is uniform in color space. However, relatively few of the faintest sources have been observed because most of the optical follow-up was done on modest-sized telescopes,

\subsection{Redshift Distribution}

The 2MASS red AGN discovered to-date span a redshift range of $0.03 \leq z \leq$ 2.52, as illustrated in Figure 2. However, this search finds predominantly low redshift objects; the median redshift of the sample is 0.22 , and only three AGN are found at $z>0.8$. Near-infrared color-selection biases the 2MASS search to low redshift objects because of the k-correction of AGN spectral energy distribution (e.g. Hyland \& Allen 1982). The predicted $J-K_{s}$ color for the radio-quiet QSO template of Elvis et al. (1994) is plotted versus redshift in Figure 2. QSOs from the optically-selected Hamburg/ESO survey (Wisotzki et al. 2000) and LBQS survey (Hewitt et al. 1995) are also shown in Figure 2, confirming the general trend and illustrating the typical scatter for an ensemble of objects. The predicted color of unobscured QSOs rapidly shifts to the blue with increasing redshift. The observed QSO colors exhibit a peak near $\mathrm{z} \sim 2$ because $\mathrm{H} \alpha$ enters the $K_{s}$ bandpass. Although 2MASS has the sensitivity to detect QSOs out to redshift $z=3$ and even higher, they are rare in our color-selected sample.

At low redshifts $(z \leq 0.5), 2 \mathrm{MASS}$ is revealing large numbers of previously unknown red AGN. Taken together with the optical/UV-selected objects, they show that the intrinsic spread in $J-K_{s}$ colors of AGN is at least a factor of two larger than previously known.

\subsection{Luminosity Distribution}

The bias against higher redshift objects means that the highest luminosity objects are underrepresented in the 2MASS red AGN sample. If the samples are limited to $z<0.4$, the 2MASS Type 1 AGN and Hamburg/ESO QSOs have a similar range of $K_{s}$-band luminosity, as shown in Figure 3. The median and RMS values of $\log h^{-2} L_{K} / L_{\odot}$ are $9.8+/-0.3$ and $9.6+/-0.5$ for the 2MASS Type 1 AGN and Hamburg/ESO QSOs, respectively. However, since $\leq 5 \%$ of the Hamburg/ESO QSOs have $J-K_{s}>2.0$, the samples are largely mutually exclusive.

\subsection{Detection in Radio, Far Infrared and X-Ray Surveys}

Approximately 50\% of the confirmed 2MASS Type 1 and 2 AGN have counterparts within 2" in the FIRST radio survey (Becker et al. 1995). Virtually all of the radio-detected sources are radio-quiet or "moderate" with $S_{1.4 G H z} / S_{K_{s}} \leq$ 1. Only $\sim 20 \%$ of the new 2MASS AGN were detected in one or more bands by IRAS, indicating that the 2MASS red AGN search does not effectively sample the extreme IR QSO population found by Low et al. (1988). Only $\sim 10 \%$ of the 2MASS AGN are detected in the ROSAT Faint Source Survey. 


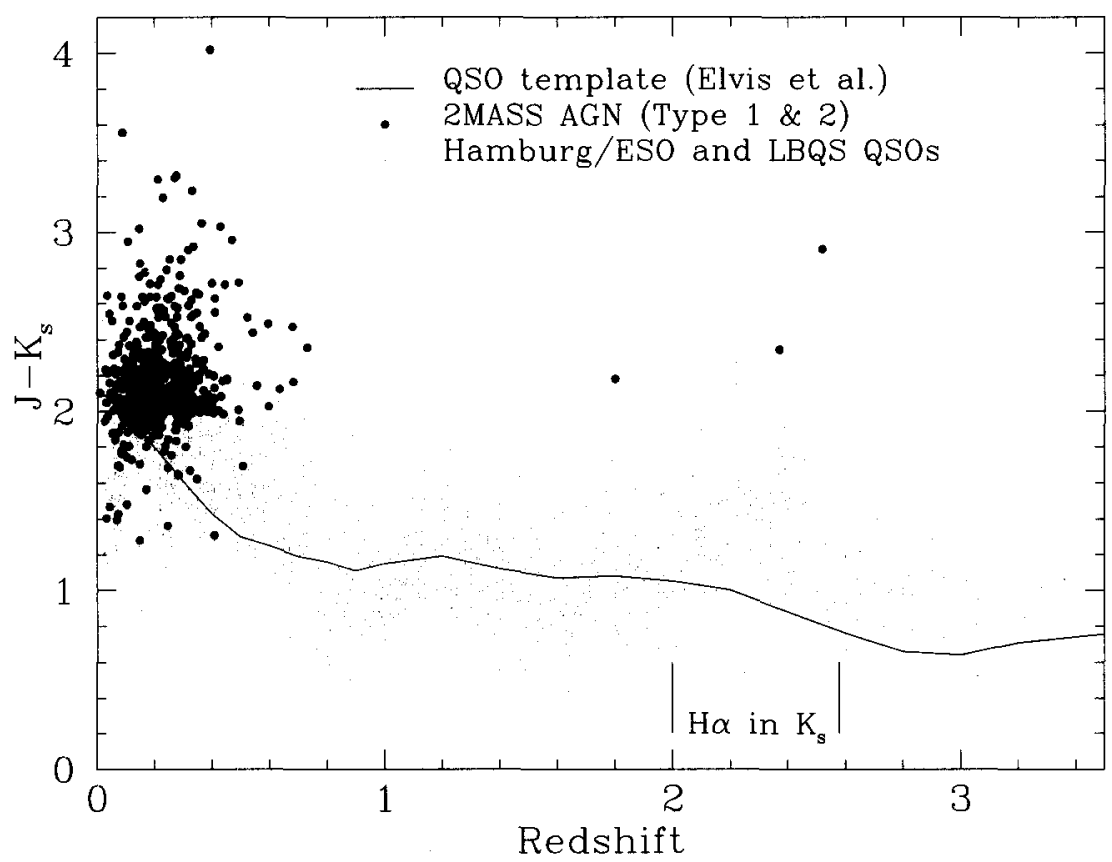

Figure 2. The 2MASS $J-K_{s}$ colors of AGN plotted a function of redshift. New 2MASS AGN are shown as large, filled circles, and optical-color selected QSO from the Hamburg/ESO and LBQS samples are shown as small points. The line shows the color predicted using the radio-quiet QSO template of Elvis et al. (1994). The redshift range in which the $\mathrm{H}$-alpha emission line enters the $K_{s}$ window and can contaminate the $J-K_{s}$ color is shown. 


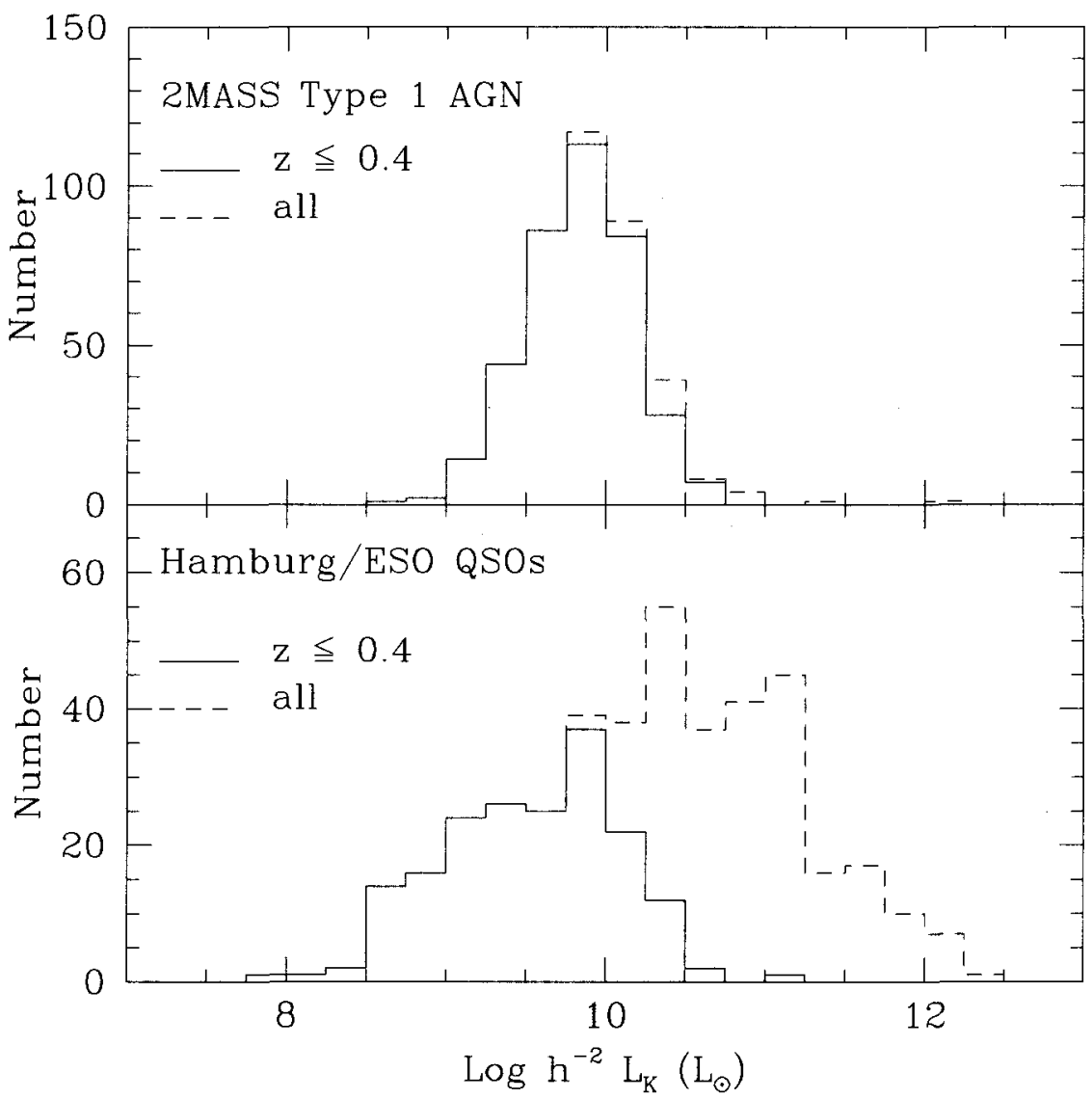

Figure 3. (top) $K_{s}$-band luminosity distributions for the 2MASS Type 1 AGN (top) and Hamburg/ESO QSOs (Wisotzki et al. 2000) (bottom). In each panel, the solid curves correspond to the subset of objects with $z \leq 0.4$, and the dashed curves to the full samples. The 2MASS color-selection finds AGN as luminous as the Hamburg/ESO survey in the local universe, but is biased against finding the most distant and luminous objects. 


\section{Discussion}

If the $69 \%$ AGN detection rate achieved for the 2MASS red AGN sample holds for the entire sample of 16,977 candidates, there will be $\sim 0.57 \mathrm{deg}^{-2}$ previously unknown Type 1 and 2 red AGN brighter than $K_{s} \leq 15.5 \mathrm{mag}(\sim 0.04 \mathrm{mJy})$ over the sky. This is equivalent to the Hamburg/ESO QSO surface density extrapolated to $B_{j} \leq 17.8 \mathrm{mag}$ (Wisotzki et al. 2000), or that at $B_{j} \leq 18.2$ mag derived from the complete spectroscopic survey of the Fornax cluster field by Meyer et al. (2001). This extrapolation is tempered by the fact that so few of the candidates fainter than $K_{s}>14.5$ have been observed to-date, though, so the behavior for the faint sources, which are in the majority, is largely unknown.

The modest sensitivity of 2MASS, and the color-selection technique we apply to the flux-limited 2MASS data set inherently biases us against finding the most heavily obscured and most distant objects. 2MASS is $>99 \%$ complete to $\mathbf{J} \leq 15.8 \mathrm{mag}$ and $K_{s} \leq 14.3 \mathrm{mag}$ at high latitudes. Sources are detected up to $\sim 1$ mag fainter than the completeness limit, but with decreasing completeness. The red color limit selects against higher redshift objects because the k-correction shifts objects to bluer $J-K_{s}$ colors with increasing redshift (Figure 2). The three-band detection requirement also biases against finding the most extreme red and possibly distant objects which are turning up in other searches. Gregg et al. (2002) and Lacy et al. (2002) discovered a number extremely red, high redshift and high luminosity QSOs by matching FIRST radio and 2MASS infrared sources. Most of these were not detected in all three 2MASS bands. Thus, the density of red, obscured AGN inferred from this search may actually underestimates the true density.

The Fornax survey field can be used to compare the volume densities of AGN from optical- and infrared-selected surveys. Meyer et al. (2001) and Drinkwater et al. (2001) found 71 QSOs in the 3.1 sq.deg. field, and seven (2.2 $\mathrm{deg}^{-2}$ ) have $z \leq 0.5$. There are eight red 2MASS AGN candidates in that region $\left(2.5 \mathrm{deg}^{-2}\right)$. This is much higher than the surface density of the full 2MASS red AGN sample, and it may be a result of the cluster in this field. Two of the red AGN candidates were identified by Meyer et al. as low redshift QSOs (FCSS J033720.9-345123, $\mathrm{z}=0.325, J-K_{s}=2.03$ and FCSS J033736.6-353335, $\mathrm{z}=0.469$, $J-K_{s}=2.19$ ). The remaining six 2 MASS candidates are fainter than the nominal optical completeness limit of survey. If $69 \%$ of the $2 \mathrm{MASS}$ candidates are AGN, the total would be $5-6$, bringing the combined number of QSOs in the $z \leq 0.5$ volume to $10-11$. Optical color-selection finds seven of them, infrared colorselection finds $5-6$, and two objects are in common to both techniques.

\subsection{IR and Optical Colors}

The near infrared color distribution of the 2MASS red AGN is consistent with 1-5 magnitudes of visual extinction being applied to the optical/UV-selected population. However, the optical-to-infrared colors are not consistent with a simple screen extinction model. Most of the 2MASS red AGN are too bright in the blue for the amount of extinction needed to produce the observed $J-K_{s}$ colors. As discussed earlier, though, host galaxy contamination of the optical nuclear light might pump up the blue brightness especially of the active nucleus is obscured. In addition, the USNOA-2.0 magnitudes tend to overestimate 
the brightness of extended sources since magnitudes were estimated using a brightness-diameter relation.

2MASS red AGN exhibit a range in $B-K_{s}$ color that spans over 7 magnitudes. The blue optical magnitudes range from $B_{j} \sim 15$ to $>21 \mathrm{mag}$. This is similar to the range observed in the radio-loud AGN samples of of Webster et al. (1995) and Francis et al. (1999). Flux-limited optical/UV searches will be biased against finding the most heavily obscured, nearby objects. Near-infrared and radio selection removes this bias and is sensitive to AGN over a much broader range of optical brightness. This may very well be the origin of the disparity in the claimed completeness of shallower optical/UV and infrared/radio surveys. The optical/UV surveys are highly complete, but down to specific optical brightness limits. IR and radio-selection finds the optically-faint AGN in the same volume.

\subsection{Polarization}

Smith et al. (2002) carried out a survey of optical broadband polarization for a representative subset of 70 new 2MASS red AGN. The 2MASS red AGN constitute one of the most highly polarized samples of radio-quiet AGN. Nine of the observed 2MASS AGN have polarization $P>3 \%$. For comparison, none of the PG QSOs have $P>3 \%$ (Berriman et al. 1990). The polarized fraction of the 2MASS AGN is comparable to the IRAS-discovered AGN (e.g. Hines et al. 1995). The intermediate type AGN (1.5-1.9) have the highest polarization fraction, and the degree of polarization correlates weakly with $J-K_{s}$ color and $K_{s}$ luminosity. However, not all of the reddest or most luminous objects are highly polarized. Galactic starlight likely dilutes the polarized emission in these objects, so the measured degrees of polarization are probably lower limits.

Spectropolarimetry has been obtained for two of the most highly polarized 2MASS red AGN by Smith et al. (2000). Both objects show strong broad lines and blue optical continua in the polarized light spectra, indicating that we are observing the broad line regions in scattered light in these objects. At least for these two objects, obscuration by dust affects the optical continua and spectra, and likely contributes to the observed red broadband colors.

\subsection{X-ray Emission}

Chandra observations of a subset of 23 (17 Type 1 and 6 Type 2) 2MASS red AGN were conducted by Wilkes et al. (2002). They found that the 2MASS AGN are generally weak X-ray emitters, with X-ray-to- $K_{s}$ flux ratios ranging from just below to over a factor of 100 lower than the low-redshift, broad-line QSO sample of Elvis et al. (1995). The reddest $\left(J-K_{s}\right)$ sources are also the weakest Xray sources. The hardness-ratios derived from the $\mathrm{X}$-ray measurements indicate that these objects have intrinsic neutral hydrogen absorbing column densities of $N_{H} \sim 10^{21-23} \mathrm{~cm}^{-2}$, larger than that of PG QSOs, but smaller than those found for samples of Seyfert 2 galaxies. However, the softest spectrum sources do not show evidence for intrinsic absorption, so at least some of the 2MASS AGN may be underluminous in X-rays.

A comparison between the amount of dust needed to explain the nearinfrared colors by reddening a "normal" QSO spectrum and that derived from the X-ray spectral hardness showed that the values of $E_{J-K} / N_{H}$ are between a factor of a few and $\sim 100$ lower than the Galactic value. This suggests that 
either the X-ray absorbing material is relatively dust free, or has unusual dust properties, or that the lines of sight towards the X-ray and infrared continuum emitting regions are different.

\section{Summary}

A simple color-based search of the 2MASS Point Source Database is revealing large numbers of previously unknown, predominantly low redshift AGN. Four times more Type 1 AGN than Type 2 AGN are found. Even though this search grows increasingly incomplete towards faint $K_{s}$ flux levels and for increasingly red colors, extrapolating the early AGN identification rate to the entire sample suggests that 2MASS will find $>10,000$ new AGN in the $|b|>30^{\circ}$ sky, $>7,000$ of which will be Type 1 .

The redshift and color distributions, source counts, and polarization and Xray properties of the 2MASS red AGN sample all suggest that we preferentially detect objects with obscured nuclei. Flux-limited optical/UV surveys will be biased against detection of such objects because they are often below the optical brightness limits. However, deeper optical surveys do begin to detect them (e.g. Meyer et al. 2001). Given the known incompleteness of this search, and the results of recent far-infrared, radio and X-ray surveys for such objects, it is likely that the obscured AGN population comprise a significant fraction of the all AGN in the universe.

\section{Acknowledgements}

This publication makes use of data products from the Two Micron All Sky Survey, which is a joint project of the University of Massachusetts and the Infrared Processing and Analysis Center/California Institute of Technology, funded by the National Aeronautics and Space Administration and the National Science Foundation. RMC and BON acknowledge the support of the Jet Propulsion Lab which is operated by the California Institute of Technology under contract to NASA.

\section{References}

Becker, R.H., Helfand, D.J., White, R.L. \& McMahon, R.A. 1995, ApJ, 450, 559 Berriman, G.B., Schmidt, G.D., West, S.C. \& Stockman, H.S. 1990, ApJS, 74, 869

Brough, S., Collins, C.A., Burke, D.J., Mann, R.G. \& Lynam, P.D. 2001, MNRAS.

Comastri, A., Setti, G., Zamorani, G., \& Hasinger, G. 1995, A\&A, 296, 1

Drinkwater, M., Engel, C., Phillips, S., Jones, S. \& Meyer, M. 2001, AAO Newlsetter, 97, 4

Elvis, M., Wilkes, B.J., McDowell, J.C., Green, R.F., Bechtold, J., Willner, S.P., Oey, S.P., Polomski, E. \& Cutri, R. 1994, ApJS, 95, 1

Francis, P.J., Whiting, M.T. \& Webster, R.L. 1999, PASA, 17, 56 
Gilli, R., Salvati, M. \& Hasinger, G. 2001, A\&A, 366, 407

Gregg, M.D., Lacy, M, White, R.L., Glikman, E., Helfand, D., Becker, R.H. \& Brotherton, M.S. 2002, ApJ, 564, 133

Hewitt, P.C., Foltz, C.B. \& Chaffee, F.H. 1995, AJ, 109, 1498

Hines, D.C., Schmidt, G.D., Smith, P.S., Cutri, R.M. \& Low, F.J. 1995, ApJ, $450, \mathrm{~L} 1$

Hyland, A.R. \& Allen, D.A. 1982, MNRAS, 199, 943

Jarrett, T.H., Chester, T., Cutri, R.M., Schneider, S., Skrutskie, M.F. \& Huchra, J.P. 2000, AJ, 119, 2498

Lacy, M., Gregg, M.D., Becker, R.H., White, R.L., Glikman, E. and Helfand, D.J. 2002, BAAS, 200, 503

Low, F.J., Cutri, R.M., Huchra, J.P. \& Kleinmann, S.G. 1988, ApJ, 327, L41

Meyer, M.J., Drinkwater, M.J., Phillips, S. \& Couch, W.J. 2001 MNRAS, 324, 343

Mushotzky, R.F., Cowie, L.L., Barger, A.J. \& Arnaud, K.A. 2000, Nature, 404, 459

Neugebauer, G., Green, R.F., Matthews, K., Schmidt, M., Soifer, B.T. \& Bennet, J. 1987, ApJS, 63, 615

Rieke, G. 1978, ApJ, 226, 550

Schmidt M. \& Green, R.F. 1986, ApJ, 305, 68

Skrutskie, M.F. et al. 1997, in "The Impact of Large Scale Near-IR Sky Surveys," eds. F. Garzon et al. (Kluwer (Netherlands), 25

Smith, P.S., Schmidt, G.D., Hines, D.C., Cutri, R.M. \& Nelson, B.O. 2000, ApJ, 545, L19.

Smith, P.S., Schmidt, G.D., Hines, D.C., Cutri, R.M. \& Nelson, B.O. 2002, ApJ, 569,23

Webster, R.L., Francis, P.J., Peterson, B.A., Drinkwater, M.J. \& Masci, F.J. 1995, Nature, 375, 469

Wisotzki, L., Christlieb, N., Bade, N., Beckmann, V., Khler, T., Vanelle, C. \& Reimers, D. 2000, A\&A, 358, 77 